\title{
Socialization of infant blue monkeys (Cercopithecus mitis stuhlmanni): Allomaternal interactions and sex differences
}

\author{
Steffen Förster ${ }^{1)} \&$ Marina Cords \\ (Department of Ecology, Evolution and Environmental Biology, Columbia University, $10^{\text {th }}$ \\ floor Schermerhorn Extension, 1200 Amsterdam Avenue, New York, NY 10027, USA)
}

(Accepted: 20 May 2005)

\begin{abstract}
Summary
An important part of the ontogeny of social mammals is the establishment of social relationships with non-mothers. Mothers may influence this socialization process, but other factors like the number and kind of potential partners available may also be important. In matrilineal societies, variation in allomaternal social experience is also likely to differ for males and females, relating to differences in their respective life histories. We investigated differences in non-maternal social relationships of 12 infant blue monkeys (Cercopithecus mitis stuhlmanni) in their first six months of life in a wild population. Based on previous findings that blue monkey infants develop spatial independence from their mothers at a relatively early age, we expected that infants would also socialize with non-maternal group members early in life. As possible determinants of variation in infant socialization, we evaluated the effects of age, sex, group composition, and timing of birth relative to the birth season. Allomaternal social relationships of infants differed between social groups, largely but not exclusively in response to differences in group composition. Adjusting for these group differences, we found that infants generally avoided spatial proximity to non-maternal adult females, whereas they associated more than expected by chance with other infants and large juvenile females, when away from their mothers. Close spatial association with other adult females and small juveniles increased when infants were near their mothers. Association with other infants decreased with proximity to the mother, apparently because peer playgroups led infants away from their mothers. Infants were spatially well integrated into the core of the group, associating with most available adult and large juvenile partners. Male infants spent more time in social play than females, and engaged in more rough-and-tumble play bouts, and in bouts of longer duration, than females. Large juvenile females regularly took care of infants, whereas adult females rarely acted as caretakers. There were no sex differences in behavior directed towards infants by non-mothers, but female infants associated more than male infants with adult
\end{abstract}

1) Corresponding author's e-mail address: sf2041@ columbia.edu

(C) Koninklijke Brill NV, Leiden, 2005

Behaviour 142, 869-896 Also available online - 
females when away from their mothers. Although sex differences in social play correspond to similar differences in the importance of fighting skills for adults, alternative explanations for the observed pattern remain plausible. Our data support the hypothesis that allomaternal care functions as infant handling practice for nulliparous females. The relatively rapid social development we observed in our subjects contrasts with the generally slow life history of the species and suggests that developmental rates during infancy and juvenility are promoted and constrained by different factors.

Keywords: blue monkeys, infants, socialization, social play, allomaternal care, sex differences.

\section{Introduction}

The development of social mammals is itself a social process, involving not only the mother but other group members as well (Hrdy, 1976; Altmann, 1980; Hofer, 1987; Nicolson, 1987; Holekamp \& Smale, 1993). In many mammals, including the primate subjects of this study, the social developmental process spans years, offering ample time for learning as experiences accumulate (Fairbanks, 1993; Berman \& Kapsalis, 1999). A prolonged period of development carries with it an increased chance that individuals will have different social experiences, which can have a variety of effects on their behavioral, physiological, and possibly reproductive performance later in life (Suomi, 1979; Tardif et al., 1984; Fairbanks, 1989; Sapolsky, 1994). The number and kind of social partners with whom a young animal interacts, as well as the type of social interactions, are important dimensions of the socialization and development process (Suomi, 1982; Champoux et al., 1991). Using observational data from a wild population of blue monkeys (Cercopithecus mitis stuhlmanni), we first investigate both normative patterns and variation in social interaction of infants with non-maternal partners, considering several proximate determinants of such variation as well as ultimate explanations for allomaternal care. We then describe sex differences in infantallomaternal interactions, and evaluate the extent to which they reflect sex differences in life history.

Many aspects of infant social behavior are well studied in primates, but broadly comparative syntheses are limited by the taxonomic bias of the study subjects. Nearly all detailed studies have been conducted on catarrhine monkeys that exhibit salient dominance hierarchies and/or that are largely terrestrial (Nicolson, 1987). Blue monkeys differ from better studied species in 
being almost completely arboreal, and in having only weakly expressed dominance relationships (Cords, 2002). Although several authors have suggested that an arboreal lifestyle slows infant development because of the added risk of injury from falls (Chalmers, 1973; Johnson \& Southwick, 1987; Karssemeijer et al., 1990), we found that blue monkey infants obtained spatial independence from their mother earlier than other similarly sized terrestrial and arboreal Old World monkeys (Förster \& Cords, 2003). Factors contributing to this acceleration of spatial independence may include a structurally complex, three-dimensional environment that provides more stimuli to infants for exploration (Rosenblum, 1974; but see Johnson \& Southwick, 1984; Hauser \& Fairbanks, 1988), or comparatively low levels of agonism within blue monkey groups (Cords, 2000a). The latter condition results in a much lower risk of infant abuse in blue monkeys compared to other Old World monkeys, which in turn may promote comparatively permissive mothering styles (Förster \& Cords, 2002). The data presented here allow us to evaluate how the relatively early spatial independence from the mother that we reported previously relates to social experiences with other group members. We examined proximity relations as well as social exchange in the form of grooming, infant handling, allomaternal care and social play. Because the social partners of a young and dependent infant might be heavily influenced by its mothers' social interactions (Nakamichi, 1996; Berman \& Kapsalis, 1999), we distinguished between periods the infant was in or out of proximity to its mother when assessing spatial relationships to other group members. We also expected that the availability of potential social partners in the group would influence the infants' social profile, and therefore explicitly searched for such effects.

Like many other catarrhine monkeys, female and male blue monkeys have very different social lives. Females remain in their natal groups for life, whereas males invariably transfer out as they approach adulthood and compete aggressively for breeding opportunities (Cords, 1987a). Examining the profile of grooming partners of immature blue monkeys (0-6 years of age), Cords (2000b) found that females had a more diverse set of grooming partners, especially young female peers, than did males. Förster \& Cords (2002) found that mothers groomed infant daughters more than sons. These differences could reflect divergent lifelong residence and social patterns of males and females. The data we present here allow us to consider sex differences in a broader set of non-maternal behavioral interactions, with a particular focus 
on the very youngest members of a social group. If any differences appeared at this early age that were related to sex differences in sub-adulthood and full adulthood, we expected that infant females would show more allomaternal social interaction than would infant males. In addition, based on the hypothesis that social play may serve as practice for motor skills needed later in life, especially fighting (Bekoff, 1988), we expected infant males to engage in rougher forms of play, and to play more often with challenging (i.e., older) partners than females. Sex differences in adult aggressive behavior characterize most Cercopithecine monkeys and many other mammals, and concomitant sex differences in social play of juveniles have been documented in many species (Fagen, 1981, 1993). It appears, however, that such sex differences in play do not generally emerge in infancy (Fagen, 1993). The relatively early spatial independence of infant blue monkeys from their mothers (Förster \& Cords, 2002) compared with some closely related species suggests that sex differences in allomaternal social interactions might also appear at an early age in this species.

\section{Methods}

\section{Data collection}

The study was conducted from November 1999 to June 2000 on three habituated groups of blue monkeys in the Kakamega Forest, western Kenya. The Kakamega Forest is the easternmost remnant of the semi-deciduous rainforest that once stretched continuously across much of central Africa (Hamilton, 1982). The study site included natural forest as well as areas of regenerating forest (after logging $\sim 50$ years previously), areas where enrichment planting of indigenous and non-indigenous trees had occurred $\sim 45$ years before, and small areas of plantation forest including both indigenous and nonindigenous species. Cords (1987b) gives a more detailed description of the site. The home range of the largest monkey group, $T_{\mathrm{W}}$, included a forestry station inhabited by humans, but this part of the range was rarely used during the study period. Groups varied in size and composition (Table 1). Two of the groups, $\mathrm{G}_{\mathrm{S}}$ and $\mathrm{G}_{\mathrm{N}}$, were newly formed, the result of a group fission during the July-October 1999 mating season. Most births in this population occur from January to March (Cords, 1987a), and a distinction was made between infants born first in each group and those born later in the birth season. 
Table 1. Group composition at the end of the study period. Age classes are defined as follows: infant $<1$ year, small juvenile $1-2$ years, medium juvenile 3-4 years, large juvenile 5-6 years, adult $>6$ years or after first birth. Note that not all infants in each group were included in this study.

\begin{tabular}{|c|c|c|c|c|c|c|c|c|c|c|}
\hline \multirow[t]{2}{*}{ Group } & \multirow[t]{2}{*}{$\begin{array}{c}\text { Adult } \\
\text { females }\end{array}$} & \multicolumn{2}{|c|}{$\begin{array}{c}\text { Large } \\
\text { juveniles }\end{array}$} & \multicolumn{2}{|c|}{$\begin{array}{l}\text { Medium } \\
\text { juveniles }\end{array}$} & \multicolumn{2}{|c|}{$\begin{array}{c}\text { Small } \\
\text { juveniles }\end{array}$} & \multicolumn{2}{|c|}{ Infants } & \multirow[t]{2}{*}{ Total } \\
\hline & & $0^{x}$ & q & $0^{x}$ & q & $0^{x}$ & q & $0^{x}$ & q & \\
\hline $\mathrm{T}_{\mathrm{W}}$ & 13 & 3 & 3 & 6 & 5 & 5 & 9 & 3 & 2 & 49 \\
\hline $\mathrm{G}_{\mathrm{S}}$ & 11 & 1 & 5 & 4 & 3 & 1 & 1 & 5 & 3 & $36^{*}$ \\
\hline $\mathrm{G}_{\mathrm{N}}$ & 8 & 1 & 0 & 4 & 1 & 4 & 3 & 1 & 1 & 23 \\
\hline
\end{tabular}

* Includes 2 unidentified large juveniles.

SF conducted focal samples of 12 infants (Table 2) for $336 \pm 58$ minutes in each two-week period for the first three months of life, and for an average of $148 \pm 15$ minutes for the second three months, totaling on average $38.5 \pm 10.3$ hours per focal subject. Given the difficulty of maintaining extended visual contact with the subjects, observations were made opportunistically, distributed as evenly as possible over each two-week time block and period of the day (7:30-11:00 hrs, 11:00-14:30 hrs, and 14:30-18:00 hrs). Behavior was scored on checksheets using both point and one-zero recording methods (Altmann, 1974). Sample intervals were 30 seconds for activity categories and 60 seconds for spatial relationships to individuals other than the mother. We scored the infant's distance from the mother at each activity point sample, and defined proximity as any distance less than 1 meter from the mother (including contact). Play behavior was recorded as one-zero for each sample interval. Play bouts were distinguished by periods of at least one 30 second interval in which no play occurred. Behavioral events of short duration ( $<30$ seconds) were recorded between sample intervals on a continuous basis (e.g., infant handling events, socio-sexual behavior). Each focal sample lasted about 75 minutes. If subjects went out of view for less than 15 minutes, we prolonged sampling accordingly, but we terminated the sample after breaks of longer duration, using the next opportunity within the same diurnal period to complete the sample. For more information on sampling procedures, see Förster \& Cords (2002). We recognized individuals by features of their faces, tails, and other body parts. The social partners of infants were categorized in five age classes (Table 1). 
Table 2. Birth date, sex and relative size of the subjects, the age up to which they were sampled, parity and relative rank of their mothers, and group membership.

\begin{tabular}{|c|c|c|c|c|c|c|c|}
\hline \multirow{2}{*}{$\begin{array}{l}\text { Mother's } \\
\text { name }\end{array}$} & \multicolumn{4}{|c|}{ Infant's characteristics } & \multicolumn{2}{|c|}{ Mother's characteristics } & \multirow[t]{2}{*}{ Group } \\
\hline & Sex & $\begin{array}{l}\text { Birth date } \\
{[\mathrm{mm} / \mathrm{dd} / \mathrm{yy}]}\end{array}$ & Season $^{1)}$ & $\operatorname{Age}^{2)}$ & Parity & $\begin{array}{l}\text { Relative } \\
\text { rank }^{3)}\end{array}$ & \\
\hline Fletcher & male & $12 / 13 / 99$ & early & 24 & multiparous & medium & $\mathrm{T}_{\mathrm{W}}$ \\
\hline Tap & male & $01 / 04 / 00$ & late & 24 & multiparous & low & $\mathrm{G}_{\mathrm{N}}$ \\
\hline Bibi & male & $01 / 10 / 00$ & early & 24 & multiparous & low & $\mathrm{G}_{\mathrm{S}}$ \\
\hline Pody & male & 02/01/00 & late & 20 & multiparous & medium & $\mathrm{G}_{\mathrm{S}}$ \\
\hline Spanner & male & 02/07/00 & late & 22 & multiparous & low & $\mathrm{T}_{\mathrm{W}}$ \\
\hline Plume & male & 02/09/00 & late & 12 & multiparous & low & $\mathrm{G}_{\mathrm{S}}$ \\
\hline Lolita & female & $11 / 16 / 99$ & early & 24 & primiparous & medium & $\mathrm{G}_{\mathrm{S}}$ \\
\hline Dingle & female & $11 / 26 / 99$ & early & 24 & primiparous & low & $\mathrm{G}_{\mathrm{N}}$ \\
\hline Bow & female & $12 / 13 / 99$ & early & 24 & primiparous & medium & $\mathrm{T}_{\mathrm{W}}$ \\
\hline Mustache & female & $01 / 30 / 00$ & late & 24 & multiparous & low & $\mathrm{G}_{\mathrm{S}}$ \\
\hline Angle & female & $02 / 04 / 00$ & late & 20 & multiparous & high & $\mathrm{G}_{\mathrm{S}}$ \\
\hline Xmas & female & $02 / 27 / 00$ & late & 20 & multiparous & high & $\mathrm{G}_{\mathrm{S}}$ \\
\hline
\end{tabular}

1) Timing of birth relative to birth season: early born infants were the first one or two infants born in each group and separated from a distinctive cohort of subsequent infants by 3 weeks or more.

${ }^{2)}$ Maximum age (in weeks) up to which infant was sampled.

${ }^{3)}$ Females in each group were assigned rank classes (low, medium, high) according to their position in the upper, middle, or lower third of the hierarchy.

\section{Data analysis}

In our analysis we used the proportion of the total number of points or intervals in which a given parameter was recorded, averaged for each infant across samples for each 2 or 4 week period. To exclude as much as possible the effect of maternal partner choice on an infant's social interaction patterns, we analyzed data on infant proximity partners in two ways, first considering all available data, and second, considering only periods when the infant was away from its mother. We present results from the restricted data set, however, only when they differed qualitatively from those from the full data set.

To account for differences in the composition of the three study groups, our analyses were based on the ratio of observed/expected times that each age/sex class interacted with the infant rather than absolute values of such 
interaction. We calculated expected values according to proportional representation in the group. Ratios less than one represent avoidance, while those greater than one represent attraction between the infant and a social partner of a given age/sex class. Because the number of infants present in each group changed over time, we calculated expected values for all age classes for each two-week period. If the number of infants changed during a given two week period because of births or deaths, we used the average number of infants across focal samples.

Behavioral time budgets were analyzed with two-factorial repeated measures analyses of variance, using age in months as the within-subject factor, and either sex, group membership, or time of birth relative to the birth season (early vs late) as a between-subject factor. Because of the requirements of this particular analysis (no missing values allowed), only 5 months of data were included in all repeated measures analyses of variance, allowing for the inclusion of a maximal number of infants $(N=11$, excluding Plume's infant, who was only sampled up to the third month). When there were interaction effects with age, we conducted follow-up one-way analysis of variance for each month (Förster \& Cords 2002 provide further detail). Sample size was thus 12 infants for the first three months and 11 infants for months 3-6 and for the entire study period. For analyses of the relationships between independent variables, we used 2-week averages from all infants in bivariate correlations if prior tests showed that there was no significant difference between individuals in the underlying measures, and one-way analysis of variance in all other cases. We used Spearman rank correlation tests when data distribution deviated significantly from a normal distribution, and Pearson correlation coefficients in all other cases. For averaging correlation coefficients across infants, z-transformations were applied to the coefficients, values weighted by sample size (Sokal \& Rohlf, 1995), and mean z-values back-transformed to give an average correlation coefficient. All tests were two-tailed. 


\section{Results}

\section{Allomaternal social behavior}

1. Spatial association with non-mothers

Although the number of infants in each group was not large, spatial association between infants and non-mothers appeared to differ between groups. The three $\mathrm{T}_{\mathrm{W}}$ infants associated most often with medium juveniles (mean $=12.7 \%$ of observation time), while the seven $\mathrm{G}_{\mathrm{S}}$ infants associated most with other infants (15\%), and the two $\mathrm{G}_{\mathrm{N}}$ infants associated about equally with adult females $(13.5 \%)$ and small juveniles $(14.6 \%)$. As interactions of infants with the adult male or large juvenile males were extremely rare, we do not present data for these age/sex classes. During periods when infants were away from their mother $(>1 \mathrm{~m})$, the same relative preferences were observed in $T_{W}$ and $G_{S}$, but in $G_{N}$ the association of infants with small juveniles exceeded that for adult females (17\% vs $9 \%)$. To determine whether these differences simply reflected between-group differences in the number of available social partners, we adjusted proximity scores by comparing observed association rates to random expectations based on proportional representation of the age/sex classes in the group. In all three groups, infants associated more than expected by chance with other infants and large juvenile females, and less than expected by chance with non-maternal adult females (Figure 1). Association with small juveniles was essentially random in $\mathrm{G}_{\mathrm{N}}$, but lower than expected by chance in both $\mathrm{G}_{\mathrm{S}}$ and $\mathrm{T}_{\mathrm{W}}$. In contrast, infants showed a very pronounced bias in their association with medium juvenile females in $T_{\mathrm{W}}$, whereas association with that age/sex class appeared to be random in $\mathrm{G}_{\mathrm{S}}$ and $\mathrm{G}_{\mathrm{N}}$. The bias towards other infants, although present in all groups, was almost twice as high in $\mathrm{G}_{\mathrm{N}}$ as in $\mathrm{G}_{\mathrm{S}}$ and $\mathrm{T}_{\mathrm{W}}$.

As expected, the identity of infants' proximity partners was related to close spatial association with the mother. The more time infants spent far away from their mothers the less they associated with other adult females (Figure 2A), and the more they associated with other infants (Figure 2B). This pattern was not surprising, given that most interactions with other infants involved social play, and there was a strong correlation between proportion of playtime and proportion of time spent out of proximity to the mother (mean correlation $r=0.82, N=12$ infants). In contrast, infants tended to be in proximity to small juveniles more when nearer their (own) mothers (Figure 2C), especially in months 3-5. Social play with small juveniles 


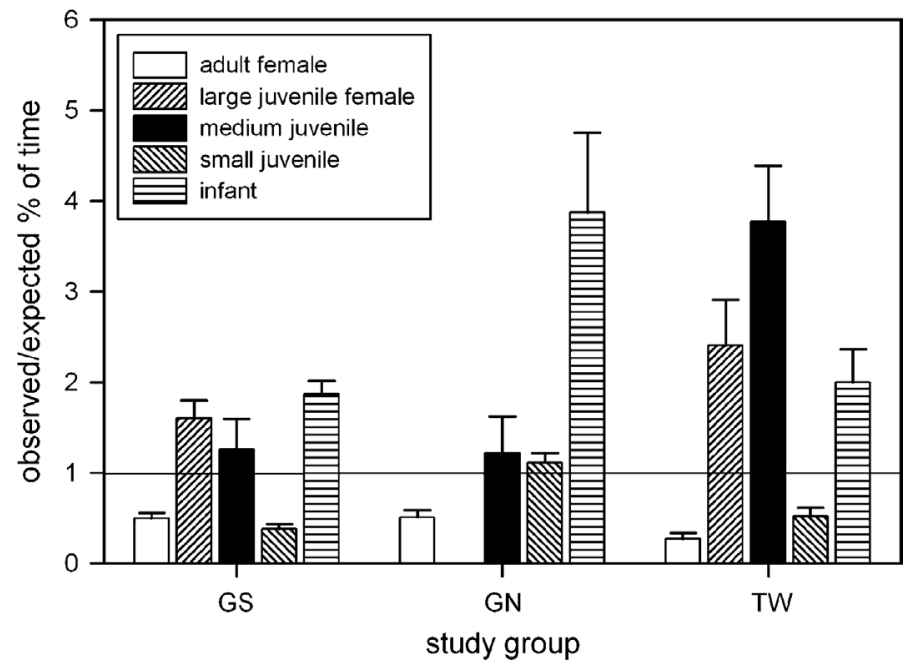

Figure 1. Ratio of observed/expected proportion of time infants spent in proximity to different non-maternal age/sex classes in each study group. There were no large juvenile females in $\mathrm{G}_{\mathrm{N}}$. Values below 1 indicate avoidance, values above 1 preference for spatial association, relative to expectations based on the proportion of each age class present in each group.

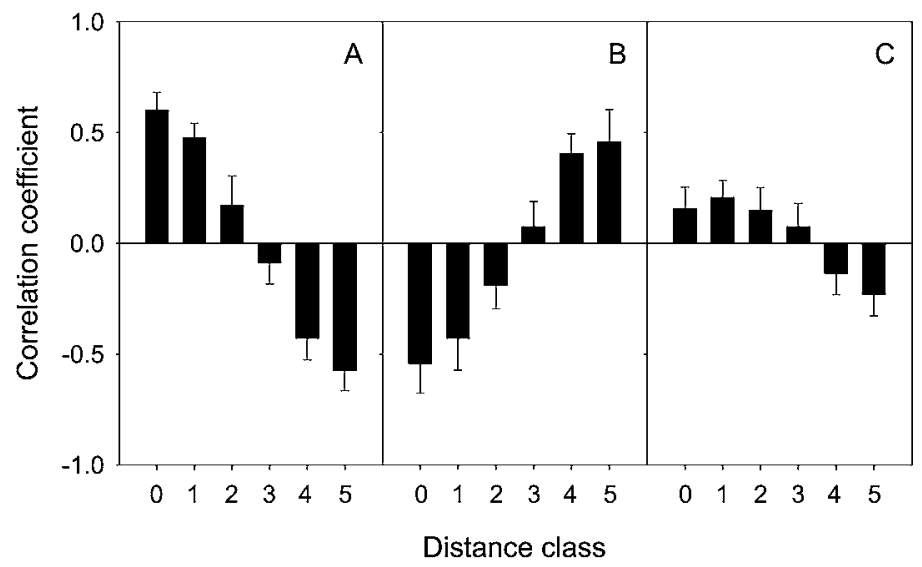

Figure 2. Correlation between time spent at a distance from the mother and time spent in spatial association (relative to random expectation) with (A) adult females, (B) infant peers, and (C) small juveniles. For each partner age-sex class, bars represent the mean ( \pm SE) of correlation coefficients calculated separately for each infant $(N=12)$ and mother-infant distance class: 0 - contact, 1 - within arms length, 2 - arms length to 1 meter, $3-1$ to 3 meters, $4-3$ to 10 meters, 5 - more than 10 meters. 
was much less frequent than play with infants (see below), and appeared not to draw infants away from their mothers. Varying spatial association with the mother did not systematically affect infants' interactions with any other age/sex class.

To evaluate the spatial integration of infants into the core of adult and subadult females of the group independently from their mother, we analyzed proximity partner identities when infants were more than one meter away from the mother. During these times, infants had a total of 3-12 nonmaternal adult females (mean $=6.7$ ) as proximity partners, which was 25 $100 \%$ (mean $=83 \%$ ) of all available adult females in their group. Similarly, infants were in proximity to 4-8 large juvenile females (mean $=6.3$ ), equaling $57-100 \%$ (mean $=82 \%$ ) of all partners in that age/sex class. Proximity to these adult and subadult female partners was not spread evenly among them, with the top 3 adult female partners accounting for an average of $67 \%$ (range: $45-100 \%$ ) of time infants spent in proximity to adult females, while the top 3 large juvenile female partners accounted for 81\% (range: 63-99\%) of proximity to that age/sex class, while infants where away from the mother.

In the first 3 months, there were differences between later- and early-born infants in the degree to which association with other infants compared to random expectations: On average, all early born infants associated with other infants less than expected by chance, whereas all later born infants associated with other infants more than expected by chance $(F=11.9, p<0.01$, $N=12$, one-way ANOVA). In fact, there was a linear relationship between the number of infants present in the group and the magnitude of the bias towards infants as proximity partners $(F=17.9, p<0.001, N=11$, repeated measures ANOVA). Although birth order was related to parity (all three primiparous mothers gave birth early in the season), parity alone had no significant effect on the association with other infants in the first three months of an infant's life. There were no effects of either timing of birth or parity on the association with other age/sex classes, and the spatial association of infants with individuals of other age/sex classes did not change over time in a way that was consistent across individuals.

\section{Infant handling and grooming}

Female group members generally showed much interest in newborn infants. Adult and large juvenile females who approached the mother would sometimes inspect the clinging infant, lifting its tail and sniffing the anogenital 
region. Other social interactions with newborn infants included smelling, touching, grooming (especially the tail), and holding. These interactions with the infant while it was on the mother were defined as non-maternal 'infant handling' and were treated separately from non-maternal 'caretaking behavior' that occurred when the infant had been taken away from the mother (see below). Most handling interactions were non-aggressive, although handlers sometimes forcefully pulled infants from the mother in an attempt to hold them. Mothers were usually intolerant of such attempts during the first weeks of an infant's life, and often threatened, bit, or chased the handler, or shielded the infant by turning away and embracing it. Each infant was handled by an average of 9 different individuals (range: 2-19), which is a minimum estimate because handlers could not be identified in all cases.

The overall proportion of intervals in which infant handling occurred was low in all study groups (mean $=1 \%$, range: $0.1-2, N=12$ ). Non-maternal infant handling was not equally common in the three study groups during the first three months ( $F=6.7, p<0.01, N=12$, one-way ANOVA). Six of the seven infants in $\mathrm{G}_{\mathrm{S}}$ were handled significantly less by all group members than all infants in $\mathrm{G}_{\mathrm{N}}(p<0.05)$ and $\mathrm{T}_{\mathrm{W}}(p<0.01$; Bonferroni post-hoc tests). The overall proportion of time each infant was handled by non-mothers decreased linearly with the time difference between its birth and the birth date of the first born infant in the group $(r=-0.94, p<0.001$, $N=12$ ). This result is consistent with a loss of interest in infants later in the season, or the distribution of handlers' attention between more infants. Adult females were the most frequent infant handlers in all groups $(46 \%$, $43 \%$, and $21 \%$ of all handling in $\mathrm{G}_{\mathrm{N}}, \mathrm{G}_{\mathrm{S}}$, and $\mathrm{T}_{\mathrm{W}}$, respectively), followed by large juvenile females (14\% and $16 \%$ of all handling in $\mathrm{G}_{\mathrm{S}}$ and $\mathrm{T}_{\mathrm{W}}$, respectively). Medium and small juveniles accounted for an average of $8 \%$ of infant handling in all groups (range: 3-13\%). After adjusting for the availability of potential infant handlers in each group, we found adult females handled infants about as often as expected by chance (Figure 3). Infant handling that occurred at rates exceeding random expectations involved large juvenile females and all infants in $\mathrm{T}_{\mathrm{W}}$ (mean: 3.4 times expected, range: 1.9-7.6), and three of the seven infants in $\mathrm{G}_{\mathrm{S}}$ (mean $=2$ times expected, range: 1.4-3.0, compared to mean $=0.4$, range: $0-0.8$ for all other infants in that group). Handling by medium juveniles, on the other hand, exceeded random expectation in 4 of 7 infants in $\mathrm{G}_{\mathrm{S}}$ (mean $=5.4$ times expected, range: 2.1-8.5 times expected, compared to mean $=0.2$, range: $0-0.5$ for other infants in 


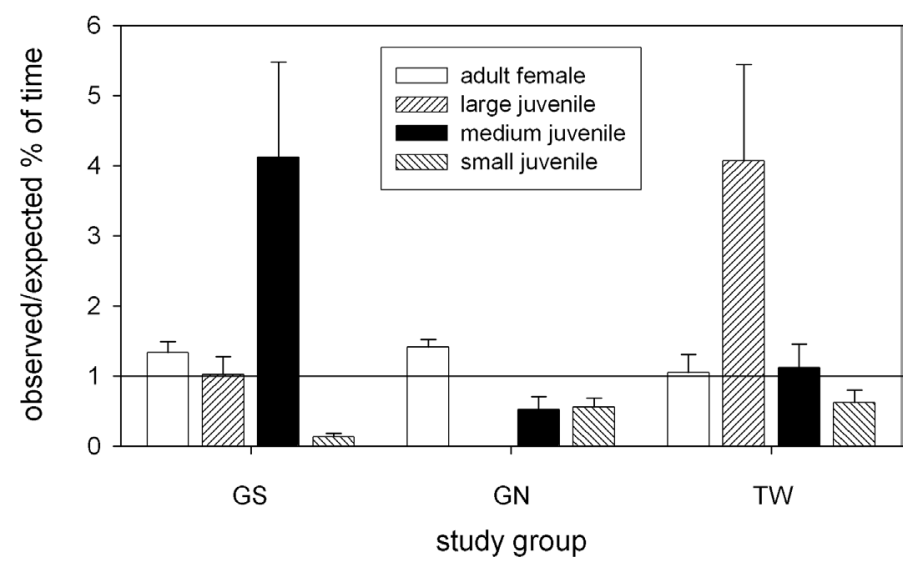

Figure 3. Infant handling (all interactions with infants while they were on the mother) by different age/sex classes (mean $\pm \mathrm{SE}$ ), and segregated by group, during the first three months of life. Shown is the ratio of the observed/expected proportion of time individuals in each age/sex class handled infants, where expected values were based on proportional representation in the group. Differences between age/sex classes were significant in all groups $\left(F=7.8, p<0.001, N=10\right.$ two-week time-blocks for $\mathrm{G}_{\mathrm{N}} ; F=6.1, p<0.01, N=24$ for $\mathrm{G}_{\mathrm{S}} ; F=4.8, p<0.01, N=13$ for $\left.\mathrm{T}_{\mathrm{W}}\right)$.

that group), but was lower than expected for both $\mathrm{G}_{\mathrm{N}}$ infants and for two of three $\mathrm{T}_{\mathrm{W}}$ infants (mean $=0.5$ times expected, range: $0.1-0.9$ ). Small juveniles were generally underrepresented as infant handlers in all groups (mean $=0.2$, range: $0-0.6$ times expected).

Grooming is the one component of handling behavior that persists throughout life in many primates. It also appears to be the most affiliative form of handling, and least likely to cause distress in the infant. We found that infants were almost always the recipients of grooming. Generally, non-mothers accounted for more infant grooming than mothers, with large and medium juveniles accounting for most of the non-maternal grooming. There was considerable variation among infants in the mean ratio of received grooming that came from non-mothers vs. mothers (range: $0.1-8, N=11$ infants, with mean values computed across all 2-week periods), which was possibly related to infant sex (see section on sex differences below). The overall amount of grooming infants received from non-mothers was not systematically related to any independent factors in our repeated measures analyses of variance, and did not differ significantly between infants. However, both infants in $\mathrm{G}_{\mathrm{N}}$ received overall more grooming from adult females than any infants in the other groups (mean $=2 \%$ and $1.5 \%$ of focal time for both $\mathrm{G}_{\mathrm{N}}$ 
infants, vs mean $=0.4 \%$, range: $0.1-0.9 \%$ in $\mathrm{G}_{\mathrm{S}}$, and mean $=0.3 \%$, range: $0.2-0.4 \%$, in $\mathrm{T}_{\mathrm{W}}$ ).

\section{Interaction with peers: social play}

First social play occurred in weeks 5-6 (on average) of an infant's life (range: weeks 3-4 to 11-12, $N=12$ ). Its onset, however, was significantly different between first born and later born infants (weeks 9-10 versus weeks 5-6, one-way ANOVA: $F=8.4, p<0.05$ ). Early social play occurred with infant peers or small juveniles and consisted of slow wrestling movements. Solitary object play was also observed, especially when no playmates were available, but was far less common than social play and usually consisted of manipulating twigs and small sticks with hands and mouth. Infants spent about $4 \%$ of their time in social play by the third month, and about $10 \%$ of their time when six months old. Although the total proportion of intervals spent in social play did not differ between groups $\left(\chi^{2}=1.8\right.$, df $=2$, $p=0.4$ ), there were significant differences among groups in the age-sex profiles of play partners. For the seven $\mathrm{G}_{\mathrm{S}}$ infants, other infants made up an overall greater proportion of play partners than for any infant in the other groups $\left(89 \pm 7 \%\right.$ of play partners in $\mathrm{G}_{\mathrm{S}}$, compared to $45 \pm 4 \%$ in $\mathrm{G}_{\mathrm{N}}$, or $52 \pm 13 \%$ in $\mathrm{T}_{\mathrm{W}} ; F=30, p<0.001, N=11$, repeated measures ANOVA and Bonferroni posthoc test). However, relative to the number of infants available as play partners in each group, there was a much stronger bias towards other infants as play partners in both $\mathrm{G}_{\mathrm{N}}$ infants compared to any infant in the other groups (11 and 17 times the expected value, vs a mean of 3.4 and 4.2 times more than expected in $\mathrm{G}_{\mathrm{S}}$ and $\mathrm{T}_{\mathrm{W}}$, respectively; $p<0.001$, Bonferroni posthoc test). For five of the seven infants in $\mathrm{G}_{\mathrm{S}}$, the proportion of small juveniles as play partners, on the other hand, was lower than for any infant in $\mathrm{G}_{\mathrm{N}}$ or $\mathrm{T}_{\mathrm{W}}\left(2 \pm 5 \%\right.$, compared to $53 \pm 3 \%$ in $\mathrm{G}_{\mathrm{N}}$, and $34 \pm 12 \%$ in $\mathrm{T}_{\mathrm{W}} ; p<0.001$ and $p<0.05$, respectively, Bonferroni posthoc test), and this result held when we accounted for group composition (mean of 0.4 times the expected value vs 1.3 and 1.1 in $\mathrm{G}_{\mathrm{N}}$ and $\mathrm{T}_{\mathrm{W}}$, respectively, $p<0.01$ ). Play with medium juveniles was never observed in $\mathrm{G}_{\mathrm{S}}$, and only rarely in the other groups ( $12 \%$ and $6 \%$ of play time in $\mathrm{G}_{\mathrm{N}}$ and $\mathrm{T}_{\mathrm{W}}$, respectively).

\section{Allomaternal caretaking}

Allomaternal care of infants was a regular occurrence in this population. It most often involved infants aged 2-5 months. There were, however, consid- 
erable inter-individual differences in the overall proportion of time an infant was carried by non-mothers (range: $0.3-8.2 \%$ ) and its distribution over time. Infant sex, social status of the mother, timing of birth or group membership had no main effects on the proportion of time infants were carried by nonmothers in our repeated measures analyses of variance. The main caretakers of infants in $\mathrm{G}_{\mathrm{S}}$ and $\mathrm{T}_{\mathrm{W}}$ were either medium or large juvenile females. Only in $\mathrm{G}_{\mathrm{N}}$ did two adult females regularly take care of an infant, but caretaking activity was generally infrequent in this group $(0.3$ and $1.2 \%$ of intervals for the two $\mathrm{G}_{\mathrm{N}}$ infants vs $0.4-10.1 \%$ in $\mathrm{G}_{\mathrm{S}}$, mean $=3.3 \%$, and $4.5-8.2 \%$ in $\mathrm{T}_{\mathrm{W}}$, mean $=5.8 \%$; differences between groups: $F=5, p<0.01, N=11$ ). Although never observed during this study, a large juvenile male was seen once in 2003 carrying the infant of its mother for a few seconds. On two occasions, adult females from $\mathrm{T}_{\mathrm{W}}$ and $\mathrm{G}_{\mathrm{S}}$ who had infants of their own carried another female's infant. In one case, a female carried another infant together with her own when crossing a gap in the canopy, after the resident adult male gave an alarm call.

All infants were carried by more than one allomother, and most allomothers cared for more than one infant during the study period. However, each caretaker seemed to prefer certain infants that were carried more than any other (Figure 4), leading to a significant deviation from an equal distribution of caretaker's activities among infants for 3 of 7 caretakers in $\mathrm{G}_{\mathrm{S}}$. Although sample size in $\mathrm{T}_{\mathrm{W}}$ was too small for meaningful statistical analysis, 4 of 8 caretakers were seen caring for only one of the three infants. We had limited opportunities to investigate the hypothesis that maternal kinship structures allocare relationships. However, we observed that infants with no older siblings were cared for by non-kin, and the two infants with older female siblings that could have acted as caretakers were never (Angle's infant and Adelaide) or not preferentially (Bibi's infant and Softeyes) cared for by their sisters (Figure 4). Indeed, the mothers in these cases appeared to restrict access to their infants by the older sisters more than by other juveniles.

Large juvenile females seemed to avoid adult females when caring for an infant: while infants were away from their mother, the more time infants spent in proximity to large juvenile females, the less time they were close to adult females ( $r=-0.36, p<0.001, N=111$ two-week averages). However, mothers may have actively left their infants with caretakers once they reached an age of about three months. We found a significant correlation between the average number of leaves initiated by the mother and the 


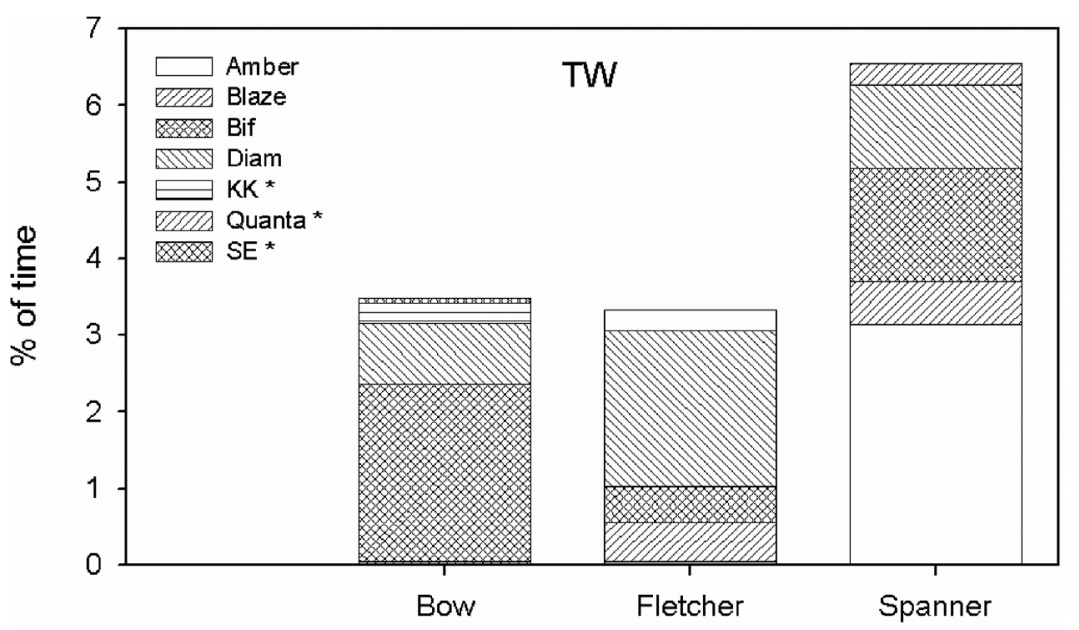

GS

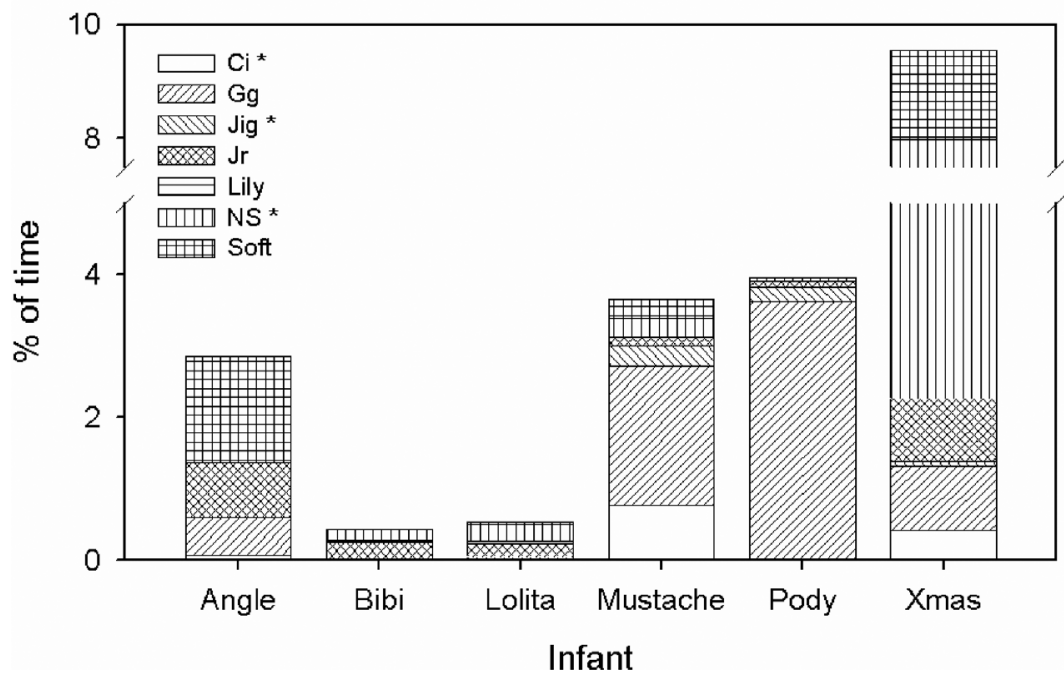

Figure 4. Distribution of allomaternal care (proportion of time) by different caretakers (represented by different shading patterns) of infants in $\mathrm{T}_{\mathrm{W}}$ and $\mathrm{G}_{\mathrm{S}}$ groups. Although all infants were cared for by more than one allomother, the majority of allomothers showed preferences for certain infants. An asterisk indicates caretakers whose efforts were nonrandomly distributed among infants $\left(\mathrm{G}_{\mathrm{S}}\right)$ or who cared only for one infant $\left(\mathrm{T}_{\mathrm{W}}\right)$. Plume's infant was excluded because it was not followed after the third month, when caretaking activity was at its peak. The exceptionally high value for Xmas's infant being carried by NS was caused by an injury the infant had during weeks 16-18, when it could not move much on its own. 
proportion of time large juvenile females were in proximity to the infant ( $r=0.37, p<0.05, N=44$ two-week periods) between 3-6 months, but not in younger infants. In addition, the more time infants were in proximity to large juvenile females, the more time they were also in proximity to other infants ( $r=0.22, p<0.05, N=111$ two-week averages), indicating that large juvenile females were often close to a number of infants simultaneously. At the age of 3-4 months, allomothers increasingly acted as a secure base for infants, protecting them against aggressive or relatively unfamiliar groupmates or during predator alarms. Juvenile caretakers sometimes also retrieved infants after they fell out of a tree, or when they were carried away or mishandled by another individual. Thus, while mothers became increasingly responsible for leaving infants with caretakers, caretakers also seemed to increase their caretaking activity towards infants as they grew older.

\section{Sex differences}

\section{Association and interaction with non-peers}

Interactions with non-peers generally did not differ between male and female infants. However, when away from their mother, male infants spent even less time in proximity to adult females than did female infants after the third month $(0.22 \pm 0.1$ times the random expectation, $N=5$ males, vs $0.53 \pm 0.3, N=6$ females, respectively, Mann-Whitney- $U=4, p<0.05$ ). Furthermore, our data suggest that there was a likely sex difference in the balance of infant grooming received from mothers and non-mothers. Five of the six male infants received less or equal grooming from their mother compared to non-mothers in each of the first six months, whereas five of the six female infants received most of their grooming from their mothers in 1-5 of the first 6 months.

\section{Interactions with peers: social play}

Male infants spent significantly more time playing than female infants throughout the study period ( $F=19.8, p<0.01, N=11)$, and played in longer bouts ( $2.5 \pm 0.4$ intervals vs $1.8 \pm 0.3, F=10, p<0.05, N=12$ infant averages). The proportion of rough-and-tumble play was significantly higher for male than for female infants $(76.2 \pm 2.0 \%$ of all intervals where play occurred, $N=5$ males, compared to $67.8 \pm 7.5 \%, N=6$ females; $F=7.7, p<0.05$ ), whereas chase-play occurred more for female infants 
$(27.9 \pm 5.9 \%$ of all intervals where play occurred, $N=6$ females, compared to $20.5 \pm 3.1 \%, N=5$ males; $F=6.3, p<0.05)$. Rough-and-tumble play bouts lasted significantly longer in male than in female infants $(2.2 \pm 0.4$ intervals vs $1.7 \pm 0.2, F=6.4, p<0.05, N=12$ infant averages), whereas there was no difference in chase play bout length between the sexes.

\section{Sexual behavior}

Infants first mounted others as early as the $12^{\text {th }}$ week of life (median: 18.5 wks, range: $12-23$ wks, $N=6$ ). Mounting was directed mainly to other infants, but occasionally to older peers. The first mounting of a medium juvenile female by an infant (male) was observed in the infant's $24^{\text {th }}$ week. Mounting of infants by other age classes was observed on three occasions (once by an adult female and twice by a medium juvenile), and was always directed to male infants. In general there was a clear sex difference in infant mounting behavior in that female infants rarely mounted ( 2 of 17 mounts by infants) but were mounted regularly (and only) by male infants ( $G=8.2$, $\mathrm{df}=1, p<0.05$, Fisher exact test). Presenting behavior occurred first in the $13^{\text {th }}$ week in a female infant, which presented in an exaggerated way to the resident male by wagging her hindquarters towards the male from a distance. In adults, such exaggerated forms of presenting seem to be submissive rather than sexual (pers. obs.). We did not see male infants present.

\section{Discussion}

\section{Allomaternal social behavior}

Individuals in social groups of primates generally do not associate with each other randomly, but establish close social ties with only a subset of group members (Cords, 1997). These familiar social relationships allow individuals to maintain a network of close spatial relationships that is critical for the protective function of group living, and for developing alliances for effective within- and between-group aggressive competition (Seyfarth \& Cheney, 1984; Harcourt \& Stewart, 1989; Chapais, 1992; van Hooff \& van Schaik, 1992). Establishing the most beneficial relationships with particular partners in a social group is thus an important process for immature group members. In matrilineal societies like those of Cercopithecine monkeys, infants need 
to be integrated into an established social network made up largely of adult females and their female offspring (Nicolson, 1987). As expected, infants in our study associated with most (82-83\%) adult and large juvenile females in their group. In fact, infants associated spatially with a higher proportion of adult females in their group than reported for the adult females themselves (Cords, 2000a), although sample size differences complicate a direct comparison. Thus, judged from the number of adult and subadult female proximity partners, infants seem to establish spatial relationships to the core of the group at a young age.

As socialization is heavily influenced by the availability of social partners and social groups can be differently composed, group membership can have a significant effect on the social development of infants (Rosenblum \& Coe, 1977; Deputte \& Quris, 1997; Pereira \& Leigh, 2003). Although our sample size was small, partner availability varied among the three social groups, and in each group different age/sex classes acted as primary proximity partners of infants. As the age/sex classes tend to interact differently with infants, group composition is likely to translate into different social experiences for infants, which may in turn affect their social competence and sociability later in life (Suomi, 1982; Champoux et al., 1991). For example, the larger cohort of same-aged peers in $\mathrm{G}_{\mathrm{S}}$ may increase the number of close social ties for these infants later in life relative to the other groups. Furthermore, the presence of a larger number of infants as preferred play partners is likely to facilitate the spatial independence of infants from their mother, because social play was more likely to occur away from the mother. Indeed, it has been suggested that social relationships established between peers may be the primary element of the socialization process with regard to non-maternal partners (Ransom \& Rowell, 1972; Suomi, 1982). Furthermore, the presence of a large number of possible caretakers in $T_{\mathrm{W}}$ and $\mathrm{G}_{\mathrm{S}}$ may integrate infants more into the established social network of these groups than in $G_{N}$.

Our data also show patterns of infant spatial association that transcend differences in partner availability. Adult females and small juveniles were generally underrepresented as proximity partners of infants (indicating avoidance), whereas infants and large juveniles were overrepresented (indicating preference). Medium juveniles showed a strong preference to associate with infants in $\mathrm{T}_{\mathrm{W}}$, but associated with infants seemingly at random in the other groups. These general patterns seem to reflect differences in the kind and frequency of social interactions between infants and these age/sex classes. 
Non-maternal adult females did not interact frequently with infants, whereas large juvenile females were frequent caretakers, and infants often played with each other. The association with medium juveniles, however, did not correspond well with the frequency of social interactions infants had with this age class. Medium juveniles handled infants much more than expected by chance in $\mathrm{G}_{\mathrm{S}}$ than in the other groups, but the spatial association with them was considerably greater than expected in $\mathrm{T}_{\mathrm{W}}$ but not $\mathrm{G}_{\mathrm{S}}$. The reasons for this inconsistency are not clear.

In addition to proximity relationships, social interactions between nonmothers and infants differed between groups. Large juvenile females played an important role in allomaternal care in $\mathrm{T}_{\mathrm{W}}$ and $\mathrm{G}_{\mathrm{S}}$, for which adult females substituted only to a limited extent in $\mathrm{G}_{\mathrm{N}}$. A lack of caretakers may affect the future development and the socialization process of infants, and it may increase the costs of infant care for the mother (Fairbanks, 1990; Stanford, 1992). Although large juvenile females regularly kidnapped infants in the first months of life, or were reluctant to return an infant to its mother, we suggest that this relationship changed to one of mutual benefit at a later stage. Whereas large juveniles seemed to actively avoid mothers when carrying a newborn infant, mothers actively moved away from infants at a later stage, even in, or possibly because of, the presence of caretakers. As indicated by previous studies, allomothering may improve the fitness of mothers (Fairbanks, 1990; Stanford, 1992), but the dangers of infant maltreatment or dehydration (Maestripieri, 1994), especially when infants are very young, may cause the initial reluctance of mothers to separate from their infants.

As in other primate species (Berman, 1982a; Loy \& Loy, 1987; Nakamichi, 1989; van Noordwijk et al., 1993), we expected blue monkey mothers to play an important role in determining the social partners of their infants, both by varying their permissiveness vis-à-vis the infant's interaction with others, and by affecting the availability of potential partners, namely those near the mother. Infants were more likely to meet adult females and small juveniles when near their mothers, and more likely to associate with infants when away from the mother. Thus, mothers seemed primarily responsible for integrating infants into the network of adult females, and it is likely that the adult females with whom infants associated were the preferred proximity partners of their mother (Nakamichi, 1996). Longitudinal study would be needed to document the persistence of these early relationships. 


\section{The function of allomaternal care}

Caretaking by non-mothers, primarily large juvenile females, was a widespread activity in our study population. Although allomaternal care of infants occurs in many primate societies (see Hrdy, 1976; Nicolson, 1987; Maestripieri, 1994; Chism, 2000, for reviews), the degree to which this behavior is expressed differs considerably from species to species. Among the cercopithecine genera, the more closely related vervets (Chlorocebus) and patas monkeys (Erythrocebus) show extensive non-maternal infant carrying (5\% vervets, $8 \%$ patas; Fairbanks, 1990; Chism, 1978), whereas macaques (Macaca) and baboons (Papio) do not (Chism, 2000). However, while overall levels of carrying are high among the guenons, blue monkeys differ from vervets and patas in the timing of this behavior and the identities of allomothers. In vervets and patas, adult females are among the most active allomothers, and allocare is highest in the first month of life, tapering off with increasing independence of infants soon thereafter. In blue monkeys, however, little allocare occurred before the infant was about 2 months old. Thereafter, allocare activity reached a well-defined peak, where about half of the infants are cared for by non-mothers for more than $10 \%$ of the time in at least one month. In contrast to patas and vervets, nulliparous females were by far the most active allomothers in blue monkeys.

Several adaptive functions of allomaternal care have been suggested, including practice for the allomother, increasing foraging efficiency of the mother or promoting the socialization of the infant (Lancaster, 1971; Hrdy, 1976; Fairbanks, 1990; Stanford, 1992; reviewed in Maestripieri, 1994). Others argue that allomaternal care is simply a by-product of selection for maternal care, as females that are highly responsive to infants make good mothers (Manson, 1999; Silk, 1999). If caretaking behavior were only a by-product of selection for appropriate maternal behavior, one would expect nulliparous and parous females to engage in similar amounts of caretaking behavior, or parous females, primed hormonally and by experience, to participate more. Contrary to these predictions, we found that nulliparous females cared for infants far more than non-maternal adults. If allomaternal care served mainly to integrate the infant into the social network of the group, female infants should be preferred as caretaking objects in a female-bonded species like the blue monkey. However we found no sex differences in the amount of caretaking received by infants. Although our study was not designed to evaluate 
benefits of allomaternal care to the mother, we found that mothers tended to leave their older infants more often when caretakers were present. This pattern suggests that mothers may indeed benefit from caretakers by being able to devote more time to foraging and/or social activities. Taken together, our results provide the strongest support for the hypothesis that allomaternal functions to prepare subadult females for their future role as mothers (Lancaster, 1972). As there was an unequal distribution of caretaking activity among the medium and large juvenile females, longitudinal studies could show whether females that are more attracted to infants as immatures would have greater success in rearing their own first offspring, as reported for vervet monkeys (Fairbanks, 1990).

Our data on allomaternal care in blue monkey infants add to our knowledge of possible causes and consequences of interspecific differences in the expression of this behavior among primates. It has been shown empirically that early allomaternal care is positively related to postnatal growth rate in non-human primates (Ross \& MacLarnon, 1995; Ross, 2003). Thus, allocare could hasten the development of independence of infants from the mother, enabling females to resume cycling earlier and increase their reproductive output. Comparing allocare patterns across cercopithecine species, Chism (2000) concluded that early allomaternal care is associated with a high rate of infant development and short birth intervals in vervets and patas monkeys, while the absence of such care in macaques and baboons co-occurs with lower rates of infant development (Chism, 2000). Our data from blue monkeys do not support this hypothesized relationship, as interbirth intervals in this species are long (mean: 29 months, range: 9.5-82 months, Chowdhury \& Cords, in prep.) despite relatively high levels of allocare. Furthermore, even species without early allocare can have birth intervals of one year under optimal conditions (Hiraiwa, 1981; van Schaik \& van Noordwijk, 1985).

\section{Sex differences}

We expected that if sex differences emerged in early infancy, females would show greater social integration than males in this female-philopatric species. Female infants did indeed associate more than male infants with adult females, especially during the second three months of life, which suggests that female infants were already more integrated than males into the social network of adult females. However, there were no sex differences in grooming 
relationships with non-maternal group members during the first six months of life. This result contrasts with maternal grooming relationships, because mothers groomed female infants more than male infants by the age of six months (Förster \& Cords, 2002). Perhaps young blue monkeys diversify their set of proximity partners earlier than their grooming partners. Cords (2000b) reported that female juveniles generally had more grooming partners than males. Greater social integration of the philopatric sex thus appears to be a gradual process, where the integration in terms of grooming relationships follows spatial integration.

Male blue monkey infants played more than female infants throughout the study period, and they engaged in more rough-and-tumble (RT) play. This sex difference has been observed in many studies of primates (Hinde \& Spencer-Booth, 1967; Baldwin \& Baldwin, 1974; Owens, 1975; Mitchell, 1979; Eaton et al., 1985; Fagen, 1993) and other social mammals (Fagen, 1981), and may be related to sex differences in the type of aggressive interactions between adults. As males in many species compete actively for access to females, more and rougher play in male infants and juveniles may be adaptive, providing males with better fighting skills than females. In blue monkeys, contact fights between males occur regularly during the mating season and can end in severe, sometimes fatal injuries (pers. obs.). Aggression in adult female blue monkeys, on the other hand, consists largely of threats, supplants, and chases; they rarely engage in contact fights (Cords, 2000b). Thus, more RT play in male infants and of chase-play in female infants could be taken as support for the hypothesis that social play provides essential and sex-appropriate motor-training (Byers \& Walker, 1995). Also in line with this hypothesis are data from species with different life histories. For example, female ring-tailed lemurs (Lemur catta) aggressively compete for social status rather than inheriting it from their mother, and female infants of this species seem to play at the same rate as male infants (Gould, 1990). In red colobus (Colobus badius), where females instead of males emigrate from their natal groups, and are faced with frequent aggression from males, female juveniles seem to play more than males (Starin, 1990). However, some studies have failed to find sex differences in play of juveniles despite adults showing the typical sex differences in aggression (e.g., Wolfheim, 1978; Raleigh et al., 1979). In one group of Japanese macaques, female infants even spent more time in RT play than male infants, although the situation was reversed in juveniles (Mori, 1974). In howler monkeys, where both males and females 
may disperse from their natal group and aggression plays a great role in acquiring dominance in both sexes (Jones, 1980), female infants were reported to play more than male infants (Zucker \& Clarke, 1992). These data are not consistent with the hypothesis that rates of play correlate with patterns of aggression in adults.

In general, there is no convincing evidence that play-fighting in juveniles actually improves fighting skills in adults (Fagen, 1981); our study cannot address this issue because it was not longitudinal. Some researchers have argued that play only superficially resembles serious fighting behavior (Pellis $\&$ Pellis, 1998) or that it is more relevant to defensive behavior than aggressive competence (Biben, 1998). We are not able to address these objections with our data, which do not allow the fine-scaled motor analysis required. It remains possible that social play has mainly short-term benefits to the players rather than long-term, delayed benefits (Chalmers \& Lockehaydon, 1984) and even that sex differences in the kind and amount of play are determined by differential exposure to pre-natal androgens (Meaney et al., 1985; Pellis, 2002) and are of little adaptive significance. Like other primates (squirrel monkeys, langurs, vervets, bonnet and rhesus macaques, hamadryas and common baboons) (Baldwin \& Baldwin, 1977), juvenile female blue monkeys withdraw from social play at a younger age than males (pers. obs.). Our data show that this withdrawal is preceded by reductions in rougher forms of play at a time when other sex differences in social interactions are still absent.

\section{Developmental rates}

As judged from proximity relationships, allomaternal care, and social play, the rate of infant social development in blue monkeys appears to equal or surpass that of other closely related species. This pattern stands in contrast to the generally slower life history of this species and a long period of juvenility (Chowdhury \& Cords 2004, in prep.). Our results suggest that infant developmental rates may not respond to the same selective pressures proposed for juvenile development (Joffe, 1997). Although rates of juvenile growth and length of the juvenile period are closely associated with adult brain weight in primates, growth rates of infants and age of weaning appear to be independent of juvenile growth rates and age at maturity (Ross, 2003), supporting the notion of two independent developmental stages. More studies of infant development in free ranging populations would help to clarify the evolutionary 
significance of intra- and interspecific variation in infant care strategies and early social development.

Quick socialization of blue monkey infants does correspond to the development of relatively early spatial independence from the mother. Blue monkey infants spent less time in contact to their mother at comparable ages than both terrestrial and arboreal primates of similar size, such as patas monkeys, rhesus and long-tailed macaques, yellow baboons, and tufted capuchins (Nicolson, 1987; Fragaszy et al., 1991; Förster \& Cords, 2003). The most apparent difference between blue monkeys and these species seems to be their low levels of within-group aggression and risk for infants. These factors appear to have a strong effect on infant behavioral development in social primates.

\section{Acknowledgements}

This research was supported by the National Science Foundation (BCS 98-08273 to MC). We are grateful to the Government of Kenya, Office of the President and Ministry of Education, Science and Technology for permission to conduct research in the Kakamega Forest, to the University of Nairobi's Zoology Department and the Institute for Primate Research (National Museums of Kenya) for local sponsorship, and to the local officers of the Forestry Department for cooperation at the field site. We thank Jim Warfield for many stimulating discussions on nonhuman primate social development, and for his comments on parts of the manuscript. Special thanks also to the late Julius A. Omondi, to Praxides Akelo, Jasper M. Kirika, Simon Mbugua, and Caroline Okoyo for their assistance and social support at the field site.

\section{References}

Altmann, J. (1974). Observational study of behavior: sampling methods. — Behaviour 49: 227-267.

Altmann, J. (1980). Baboon mothers and infants. — Harvard University Press, Cambridge.

Baldwin, J. \& Baldwin, J. (1977). The role of learning phenomena in the ontogeny of exploration and play. - In: Primate bio-social development: biological, social, and ecological determinants (Chevalier-Skolnikoff, S. \& Poirier, F.E., eds). Garland Publishing, Inc., New York, p. 343-406.

Baldwin, J.D. \& Baldwin, J.I. (1974). Exploration and social play in squirrel monkeys (Saimiri). - Am. Zool. 14: 303-315.

Bekoff, M. (1988). Motor training and physical fitness: possible short- and long-term influences on the development of individual differences in behavior. - Dev. Psychobiol. 21: 601-612.

Berman, C.M. (1982). The ontogeny of social relationships with group companions among free-ranging infant rhesus monkeys I. Social networks and differentiation. - Anim. Behav. 30: 149-162. 
Berman, C.M. \& Kapsalis, E. (1999). Development of kin bias among rhesus monkeys: Maternal transmission or individual learning? - Anim. Behav. 58: 883-894.

Biben, M. (1998). Squirrel monkey playfighting: making the case for a congitive training function of play. - In: Animal play (Bekoff, M. \& Byers, J.A., eds). Cambridge University Press, Cambridge, p. 161-182.

Byers, J.A. \& Walker, C. (1995). Refining the motor training hypothesis for the evolution of play. - Am. Nat. 146: 25-40.

Chalmers, N.R. (1973). Differences in behaviour between some arboreal and terrestrial species of African monkeys. - In: Comparative ecology and behaviour of primates (Michaelm, R.P. \& Crook, J.H., eds). Academic Press, London.

Chalmers, N.R. \& Lockehaydon, J. (1984). Correlations among measures of playfulness and skillfulness in captive common marmosets (Callithrix jacchus jacchus). - Dev. Psychobiol. 17: 191-208.

Champoux, M., Metz, B. \& Suomi, S.J. (1991). Behavior of nursery/peer-reared and motherreared rhesus monkeys from birth through 2 years of age. — Primates 32: 509-514.

Chapais, B. (1992). The role of alliances in social inheritance of rank among female primates. - In: Coalitions and alliances in humans and other animals (Harcourt, A.H. \& de Waal, F.B.M., eds). Oxford University Press, Oxford, p. 29-60.

Chism, J. (1978). Relationships between patas infants and group members other than the mother. - Recent Adv. Primatol. 1: 173-176.

Chism, J. (2000). Allocare patterns among cercopithecines. — Folia Primatol. 71: 55-66.

Cords, M. (1987a). Forest guenons and patas monkeys: male-male competition in one-male groups. - In: Primate societies (Smuts, B.B., Cheney, D.L., Seyfarth, R.M., Wrangham, R.W. \& Struhsaker, T.T., eds). University of Chicago Press, Chicago, p. 98-111.

Cords, M. (1987b). Mixed-species association of Cercopithecus monkeys in the Kakamega forest, Kenya. - University of California Press, Berkeley.

Cords, M. (1997). Friendship, alliances, reciprocity and repair. — In: Machiavellian intelligence II: Extensions and evaluations (Whiten, A. \& Byrne, R., eds). Cambridge University Press, Cambridge.

Cords, M. (2000a). Agonistic and affiliative relationships in a blue monkey group. - In: Old World monkeys (Whitehead, P.F. \& Jolly, C.J., eds). Cambridge University Press, Cambridge, p. 453-479.

Cords, M. (2000b). Grooming partners of immature blue monkeys (Cercopithecus mitis) in the Kakamega Forest, Kenya. - Int. J. Primatol. 21: 239-254.

Cords, M. (2002). Friendship among adult female blue monkeys (Cercopithecus mitis). Behaviour 139: 291-314.

Deputte, B.L. \& Quris, R. (1997). Socialization processes in primates: use of multivariate analyses II: Influence of sex on social development of captive rhesus monkeys. Behav. Proces. 40: 85-96.

Eaton, G.G., Johnson, D.F., Glick, B.B. \& Worlein, J.M. (1985). Development in Japanese macaques (Macaca fusca): Sexually dimorphic behavior during the first year of life. Primates 26: 238-248

Fagen, R. (1981). Animal play behavior. — Oxford Press, New York.

Fagen, R. (1993). Primate juveniles and primate play. — In: Juvenile primates: Life history, development, and behavior (Pereira, M.E. \& Fairbanks, L.A., eds). Oxford University Press, New York, p. 182-196. 
Fairbanks, L.A. (1989). Early experience and cross-generational continuity of mother-infant contact in vervet monkeys. - Dev. Psychobiol. 22: 669-681.

Fairbanks, L.A. (1990). Reciprocal benefits of allomothering for female vervet monkeys. Anim. Behav. 40: 553-562.

Fairbanks, L.A. (1993). Juvenile vervet monkeys: establishing relationships and practicing skills for the future. - In: Juvenile primates (Pereira, M.E. \& Fairbanks, L.A., eds). Oxford University Press, New York, p. 211-227.

Förster, S. \& Cords, M. (2002). Development of mother-infant relationships and infant behavior in wild blue monkeys (Cercopithecus mitis stuhlmanni). - In: The guenons: Diversity and adaptation in African monkeys (Glenn, M.E. \& Cords, M., eds). PlenumKluwer, New York, p. 245-272.

Förster, S. \& Cords, M. (2003). Arboreality and infant behavioral development: new data from wild blue monkeys. - Am. J. Phys. Anthropol. S 36: 95-96.

Fragaszy, D.M., Baer, J. \& Adamscurtis, L. (1991). Behavioral development and maternal care in tufted capuchins (Cebus apella) and squirrel monkeys (Saimiri sciureus) from birth through 7 months. - Dev. Psychobiol. 24: 375.

Gould, L. (1990). The social development of free ranging infant Lemur catta at Berenty reserve, Madagascar. — Int. J. Primatol. 11: 297-318.

Hamilton, A.C. (1982). Environmental history of East Africa. - Academic Press, London.

Harcourt, A.H. \& Stewart, K.J. (1989). Functions of alliances in contests within wild gorilla groups. - Behaviour 109: 176-190.

Hauser, M.H. \& Fairbanks, L.A. (1988). Mother-offspring conflict in vervet monkeys: variation in response to ecological conditions. - Anim. Behav. 36: 802-813.

Hinde, R.A. \& Spencer-Booth, Y. (1967). The behaviour of socially living rhesus monkeys in their first two and a half years. - Anim. Behav. 15: 169-196.

Hiraiwa, M. (1981). Maternal and alloparental care in a troop of free-ranging Japanese monkeys. - Primates 22: 309-329.

Hofer, M.A. (1987). Early social relationships: a psychobiologist's view. — Child Devel. 58: 633-647.

Holekamp, K.E. \& Smale, L. (1993). Ontogeny of dominance in free-living spotted hyaenas: juvenile rank relations with other immature individuals. - Anim. Behav. 46: 451-466.

van Hooff, J. \& van Schaik, C. (1992). Cooperation in competition: the ecology of primate bonds. - In: Coalitions and alliances in humans and other animals (Harcourt, A.H. \& Waal, F.B.M.d., eds). Oxford University Press, Oxford.

Hrdy, S.B. (1976). Care and exploitation of nonhuman primate infants by conspecifics other than mother. - Adv. Study Behav. 6: 101-158.

Joffe, T.H. (1997). Social pressures have selected for an extended juvenile period in primates. — J. Human Evol. 32: 593-605.

Johnson, R.L. \& Southwick, C.H. (1984). Structural diversity and mother-infant relations among rhesus monkeys in India and Nepal. — Folia Primatol. 43: 198-215.

Johnson, R.L. \& Southwick, C.H. (1987). Ecological constraints on the development of infant independence in rhesus. - Am. J. Primatol. 13: 103-118.

Jones, C.B. (1980). The functions of status in the mantled howler monkey, Alouatta palliata Gray: intraspecific competition for group and membership in a folivourous neotropical primate. - Primates 21: 389-405.

Karssemeijer, G.J., Vos, D.R. \& van Hoof, J.A.R.A.M. (1990). The effect of some nonsocial factors on mother-infant contact in long-tailed macaques (Macaca fascicularis). - Behaviour 113: 272-291. 
Lancaster, J.B. (1971). Play-mothering: The relations between juvenile females and young infants among free-ranging vervet monkeys (Cercpothecus aethipos). — Folia Primatol. 13: 161-182.

Lancaster, J.B. (1972). Play-mothering: The relations between juvenile females and young infants among free-ranging vervet monkeys. — In: Primate socialization (Poirier, F.E., ed.). Random House, New York, p. 83-104.

Loy, K.M. \& Loy, J. (1987). Sexual differences in early social development among captive patas monkeys. - In: Comparative behavior of African monkeys (Zucker, E.L., ed.). Alan R. Liss, Inc., New York, p. 23-37.

Maestripieri, D. (1994). Social structure, infant handling, and mothering styles in groupliving Old World monkeys. — Int. J. Primatol. 15: 531-553.

Manson, J.H. (1999). Infant handling in wild Cebus capucinus: testing bonds between females? - Anim. Behav. 57: 911-921.

Meaney, M.J., Stewart, J. \& Beatty, W.W. (1985). Sex differences in social play: the socialization of sex roles. - Adv. Study Behav. 15: 1-58.

Mitchell, G. (1979). Behavioral sex differences in nonhuman primates. — Van Nostrand Reinhold, New York.

Mori, U. (1974). The inter-individual relationships observed in the social play of the young Japanese monkeys of the natural troop of Koshima Islet. - J. Anthropol. Soc. Nippon 82: 303-318.

Nakamichi, M. (1989). Sex differences in social development during the first 4 years in a free-ranging group of Japanese monkeys, Macaca fuscata. - Anim. Behav. 38: 737748.

Nakamichi, M. (1996). Proximity relationships within a birth cohort of immature Japanese monkeys (Macaca fuscata) in a free-ranging group during the first four years of life. Am. J. Primatol. 40: 315-325.

Nicolson, N.A. (1987). Infants, mothers, and other females. — In: Primate societies (Smuts, B.B., Cheney, D.L., Seyfarth, R.M., Wrangham, R.W. \& Struhsaker, T.T., eds). University of Chicago Press, Chicago, p. 330-342.

van Noordwijk, M.A., Hemelrijk, C.K., Herremans, L.A.M. \& Sterck, E.H.M. (1993). Spatial position and behavioral sex differences in juvenile long-tailed macaques. — In: Juvenile primates (Pereira, M.E. \& Fairbanks, L.A., eds). Oxford University Press, New York, p. 77-85.

Owens, N.W. (1975). Social play behaviour in free-living baboons, Papio anubis. - Anim. Behav. 23: 387-408.

Pellis, S.M. (2002). Sex differences in play fighting revisited: Traditional and nontraditional mechanisms of sexual differentiation in rats. - Arch. Sex. Behav. 31: 17-26.

Pellis, S.M. \& Pellis, V.C. (1998). The structure-function interface in the analysis of play fighting. - In: Animal play (Bekoff, M. \& Byers, J.A., eds). Cambridge University Press, Cambridge, p. 115-140.

Pereira, M.E. \& Leigh, S.R. (2003). Modes of primate development. - In: Primate life histories and socioecology (Kappeler, P.M. \& Pereira, M.E., eds). University of Chicago Press, Chicago, p. 149-176.

Raleigh, M.J., Flannery, J.W. \& Ervin, F.R. (1979). Sex differences in behavior among juvenile vervet monkeys (Cercopithecus aethiops sabaeus). - Beh. Neur. Biol. 26: 455465. 
Ransom, T.W. \& Rowell, T.E. (1972). Early social development of feral baboons. - In: Primate socialization (Poirier, F.E., ed.). Random House, Inc., New York, p. 105-144.

Rosenblum, L.A. (1974). Sex differences, environmental complexity, and mother-infant relations. - Arch. Sex. Behav, 3: 117-128.

Rosenblum, L.A. \& Coe, C.L. (1977). The influence of social structure on squirrel monkey socialization. - In: Primate biosocial development: Biological, social, and ecological determinants (Chevalier-Skolnikoff, S. \& Poirier, F.E., eds). Garland Publ. Inc., New York, p. 479-500.

Ross, C. (2003). Life history, infant care strategies, and brain size in primates. — In: Primate life histories and socioecology (Kappeler, P.M. \& Pereira, M.E., eds). University of Chicago Press, Chicago, p. 266-284.

Ross, C. \& MacLarnon, A. (1995). Ecological and social correlates of maternal expenditure on infant growth in haplorhine primates. - In: Motherhood in human and nonhuman primates (Pryce, C.R., Martin, R.D. \& Skuse, D., eds). Karger, Basel, p. 37-46.

Sapolsky, R.M. (1994). Individual differences and the stress response. - Semin. Neurosci. 6: 261-269.

van Schaik, C.P. \& van Noordwijk, M.A. (1985). Interannual variability in fruit abundance and the reproductive seasonality in Sumatran long-tailed macaques (Macaca fascicularis). - J. Zool. 206: 533-549.

Seyfarth, R.M. \& Cheney, D. (1984). Grooming, alliances and reciprocal altruism in vervet monkeys. - Nature 308: 541-543.

Silk, J.B. (1999). Why are infants so attractive to others? The form and function of infant handling in bonnet macaques. - Anim. Behav. 57: 1021-1032.

Sokal, R.R. \& Rohlf, F.J. (1995). Biometry. - W. H. Freeman and Company, New York.

Stanford, C.B. (1992). Costs and benefits of allomothering in wild capped langurs (Presbytis pileata). - Behav. Ecol. Sociobiol. 30: 29-34.

Starin, E.D. (1990). Object manipulation by wild red colobus monkeys living in the Abuko Nature Reserve, The Gambia. - Primates 31: 385-391.

Suomi, S.J. (1979). Differential development of various social relationships by rhesus monkey infants. - In: The child and its family. Plenum Press, New York, p. 219-244.

Suomi, S.J. (1982). The development of social competence by rhesus monkeys. - Annali dell'Istituto Superiore di Sanità 18: 193-202.

Tardif, S.D., Richter, C.B. \& Carson, R.L. (1984). Effects of sibling rearing experience on future reproductive success in two species of Callitrichidae. - Am. J. Primatol. 6: 377380.

Wolfheim, J.H. (1978). Sex differences in behaviour in a group of captive juvenile talapoin monkeys (Miopithecus talapoin). - Behaviour 63: 110-128.

Zucker, E. \& Clarke, M. (1992). Developmental and comparative aspects of social play mantled howling monkeys in Costa Rica. - Behaviour 123: 144-171. 